ÉGYPTE monde arabe

\section{Égypte/Monde arabe}

$12 \mid 2015$

Evolution des systèmes médiatiques après les révoltes arabes

\title{
Des « publics » radicaux et polarisés face à la disparition des médias libres en Egypte
}

\section{Kai Hafez}

\section{CpenEdition}

\section{Journals}

Édition électronique

URL : https://journals.openedition.org/ema/3384

DOI : 10.4000/ema.3384

ISSN : 2090-7273

Cet article est une traduction de :

Radically Polarized Publics and the Demise of Media Freedom in Egypt - URL : https://

journals.openedition.org/ema/3397 [en]

Éditeur

CEDEJ - Centre d'études et de documentation économiques juridiques et sociales

Édition imprimée

Date de publication : 25 mars 2015

Pagination : 51-65

ISBN : 9782905838858

ISSN : $1110-5097$

Référence électronique

Kai Hafez, « Des « publics » radicaux et polarisés face à la disparition des médias libres en Egypte », Égypte/Monde arabe [En ligne], 12 | 2015, mis en ligne le 25 mars 2015, consulté le 07 juillet 2022. URL : http://journals.openedition.org/ema/3384 ; DOI : https://doi.org/10.4000/ema.3384 


\section{KaI HafeZ}

\section{DES «PUBLICS » RADICAUX ET POLARI- SÉS FACE À LA DISPARITION DES MÉDIAS LIBRES EN EGYPTE}

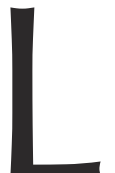

a démocratie a besoin d'une sphère publique. Il existe de nombreuses théories sur le sujet. Une des plus connues, celle d'Habermas, suggère que grâce à la délibération rationnelle entre les citoyens d'une part, et entre les citoyens et l'Etat, d'autre part, les sociétés modernes interagissent et résolvent leurs problèmes ${ }^{1}$. La légitimité dans les démocraties n'est pas seulement garantie par les élections, car les citoyens doivent aussi s'impliquer dans l'espace public et soutenir leurs élus.

La liberté des médias est un prérequis de ce processus, puisqu'elle rend possible la discussion publique. A cœur de toute démocratie, on trouve donc des élections libres, le droit de se rassembler, des normes en matière de droits humains ainsi que la liberté d'opinion et de presse.

Cette thèse soulève, cependant, plusieurs difficultés. Habermas lui-même les a appelées les " changements structurels » de la sphère publique idéale. Les médias de masse jouent un rôle crucial de médiateurs entre les citoyens, mais ne disposent d'aucun mandat démocratique et agissent très souvent comme les agents d'intérêts politiques ou capitalistes particuliers. Edward S. Herman et Noam Chomsky ont appellé cela la « fabrication du consentement $»^{2}$. D'autres théoriciens néo-marxistes comme Antonio Gramsci ont mis en avant l'existence d'une hégémonie dans la sphère publique ${ }^{3}$. Les féministes ont pour leur part critiqué Habermas pour sa croyance naïve en un

1. Habermas, 1989.

2. Herman et Chomsky, 1988.

3. Gramsci,1971. 
public rationnel, alors que, selon elles, celui-ci serait surtoutémotionnel. Chantal Mouffe et Ernesto Laclau suggèrent que la décentralisation de la sphère publique à travers Internet est un ingrédient nécessaire pour susciter une communication post-démocratique. Ils évoquent de multiples petites sphères publiques, plutôt qu'une seule sphère intégrée au plan national ${ }^{4}$.

Qu'est ce que tout cela a à voir avec l'Egypte? L'Egypte n'est certainement pas un Etat post-démocratique, pas même un Etat démocratique. Le "Printemps arabe " et la série d'élections qui ont suivi au cours des deux dernières années ont certes donné l'illusion de son existence, mais la démocratie électorale n'a jamais été pleinement atteinte ni consolidée. Tout d'abord, il y eut un parlement sans président, puis un président sans parlement - et, du coup d'Etat militaire de juillet 2013 jusqu'à l'élection du général al-Sissi en juin 2014, il n'y a eu ni président, ni parlement élus. Le président Morsi a été destitué et les Frères Musulmans bannis. En matière de liberté des médias, la situation est bien pire que celle qui prévalait sous Moubarak - celle-ci garantissait au moins une forme d'autocratie libérale, dans laquelle on pouvait exercer une critique, y compris à l'égard du gouvernement. Le coup $d^{\prime}$ Etat militaire ressemble davantage à une contre-révolution et à un retour aux années Nasser dans le rapport du pouvoir aux médias. Le mouvement populaire " Tamarrud » contre Morsi a été récupéré par les vieilles élites, et l' «Etat profond » laissé par Moubarak a pu revenir au pouvoir. L'avenir dira si la démocratie a toujours une chance en Egypte.

Cette situation est triste. Deux ans plus tôt, un véritable mouvement social avait été à l'origine d'un événement historique en mettant à bas une dictature en Egypte. Le mouvement social était le chaînon manquant qui a su rassembler les sécularistes, les islamistes et différentes forces sociales, chacune d'entre elles étant trop faible pour renverser à elle seule le régime. Il faut rappeler une situation qui semble presque absurde aujourd'hui : en février et mars 2011, des gens issus de tous les segments de la société - sécularistes, salafistes et Frères musulmans - protestaient ensemble sur la place Tahrir et dans le reste du pays. Le Printemps arabe en Egypte n'était pas une "Révolution Facebook ", même s'il a en partie été rendu possible par les nouveaux médias Internet 2.0 et les chaînes satellitaires transnationales comme Al-Jazeera, qui diffusaient en direct, 24 heures sur 24, les nouvelles de la révolution. La sphère publique ne se limitait certainement pas aux médias électroniques, les interventions orales, dans la rue, jouaient aussi un rôle important.

4. Laclau et Mouffe, 2001. 
Comment expliquer que la sphère publique pluraliste qui existait en 2011 et 2012 se soit effondrée pour devenir ce que nous connaissons aujourd'hui ? Les chaînes de télévision anciennement critiques telles que CBS, Dream TV, Al-Nahar TV, Tahrir TV, Mehwar, Sada El Balad, QahiraWalNas ou encore ONTV, relaient toutes aujourd'hui la version officielle des événements telle que présentée par le Conseil Supérieur des Forces Armées (CSFA). II en est plus ou moins de même concernant les journaux. Après le coup d'Etat contre Morsi, un encadré "luttons contre le terrorisme » est venu orner les écrans de télévision pour réduire les Frères Musulmans à une organisation terroriste. Les massacres perpétrés par l'armée contre les sympathisants des Frères Musulmans ont été minimisés ; seules étaient commentées les attaques contre les églises, alors même que les manifestations des Frères Musulmans contre le CSFA étaient majoritairement pacifiques. Depuis le coup de force, la ligne politique des médias d'Etat a effectué un tournant à 180 degrés: autrefois derrière Moubarak, leurs représentants ont soutenu Morsi, et apportent maintenant un soutien sans réserve à la direction militaire du pays. Le CSFA a fermé les médias islamistes pour mettre en place une censure bien plus stricte que celle qui existait sous Morsi. Pour la première fois peut-être dans l'histoire, les associations internationales de journalisme ont vivement critiqué un coup d'Etat contre un gouvernement islamiste, alors qu'auparavant, elles les soutenaient de manière quasi-unanime, comme lors des élections algériennes de 1991.5

Pour comprendre cette situation, nous devons analyser les structures de fonctionnement du système médiatique égyptien. Pour cela, les comparaisons internationales sont utiles car ce qui s'est passé en Egypte - la désintégration d'une sphère publique autrefois vibrante n'est pas nouveau dans un processus de transformation démocratique. La vulnérabilité des jeunes démocraties constitue presque une caractéristique universelle dans l'histoire. Deux spécialistes célèbres des médias, Daniel Hallin et Paolo Mancini, ont identifié quatre dimensions importantes pour l'analyse comparée des médias dans les démocraties, en insistant sur le type de relations entre $1 /$ l'Etat et les médias, 2/ la société civile et les médias, 3/ l'économie et les médias, 4/ la profession journalistique et les médias ${ }^{6}$. Ces dimensions couvrent la plupart des champs de la théorie des systèmes, ainsi que les interactions des médias avec d'autres sous-systèmes, comme le système politique et le système économique, ou d'autres environnements (à I'instar de la " société civile ») plus dispersés et moins organisés. Dans les démocraties, la question n'est pas seulement d'évaluer le degré

5. Hafez,1996.

6. Hallin et Mancini, 2004. 
de liberté que l'Etat laisse aux médias, mais également de savoir si les médias contribuent au développement social, économique et professionnel.

Bien sûr, la typologie d'Hallin et Mancini a été conçue pour les systèmes médiatiques d'Europe et d'Amérique du Nord. La question est de savoir dans quelle mesure de tels modèles peuvent s'appliquer, par exemple, au monde arabe ? Certains ont affirmé que l'influence de la religion a été négligée ${ }^{7}$. Cette observation est partiellement exacte, car la religion, et l'islam en particulier, joue un rôle plus grand dans la vie politique arabe moderne que la religion dans la plupart des pays occidentaux. Cependant, ce serait une erreur de présupposer une différence essentielle entre les pays occidentaux et moyen-orientaux en raison du rôle de l'islam. Les expériences arabes, et l'expérience égyptienne en particulier, ne peuvent pas être complètement coupées du précédent occidental en matière de presse et de médias ${ }^{8}$. Par exemple, aux Etats-Unis, la portée du premier amendement de la Constitution, qui garantit la liberté d'opinion et des médias, a été fréquemment restreinte sur des bases religieuses ${ }^{9}$. Hallin et Mancini ont aussi insisté sur le fait que la religion avait joué un rôle décisif dans l'histoire des médias européens, puisque que c'est la compétition entre catholiques et protestants plus qu'autre chose, qui a déclenché la propagation du monde de l'écrit, des journaux et des pamphlets. En me référant à Hallin et Mancini, j'essaierai de trouver un moyen terme entre essentialisme et eurocentrisme, en appliquant leur typologie de base tout en prenant en considération la religion et d'autres facteurs caractéristiques de la situation égyptienne actuelle.

\section{LES MÉDIAS ÉGYPTIENS ET L’ETAT}

Depuis la révolution de 1952 et au cours des soixante dernières années, l'Egypte a connu sur le long terme une orientation de fond de plus en plus favorable à la liberté des médias - tendance observable de la présidence de Gamal Abd al-Nasser jusqu'au coup d'Etat militaire de juillet 2013.

Dans l'ensemble, les dernières années d'Hosni Moubarak peuvent être caractérisées comme une " autocratie libérale ", dans laquelle il était possible de critiquer le gouvernement, même si plusieurs lignes rouges ne devaient toutefois pas être franchies. Pendant le Printemps

7. Khamis,2009.

8. Hafez, 2010.

9. Heins, 1993. 
arabe égyptien de 2011, des médias privés tels que ONTV, Dream TV ou le journal Al-Masry Al-Youm ont soutenu le soulèvement populaire, de même que les médias transnationaux telles que la chaîne qatarie AlJazeera. La liberté des médias en Egypte n'a jamais été aussi importante qu'en 2011. Les problèmes sont cependant vite réapparus lorsque, la même année, le gouvernement du CSFA a repris le contrôle du processus politique. L'armée a engagé des poursuites contre plusieurs journalistes et les graffitis critiques à l'égard de l'armée étaient régulièrement effacés. Cependant, il y avait toujours une dualité entre les médias contrôlés par l'Etat et les médias privés, critiques du pouvoir. Plus de 20 nouvelles chaînes de télévision ont été crées à cette période. Nile TV, ONTV, Al-Tahrir TV, Al-Nahar TV ou Masr 25 ont ainsi couvert les brutalités policières ou encore le massacre de Port Saïd.

La principale caractéristique de l'ère du président Mohammed Morsi a été la stagnation en matière de liberté des médias. Son gouvernement n'a pas introduit de nouveaux obstacles au sein du système médiatique, et la liberté des médias privés a persisté. Beaucoup de médias islamistes ont certes vu le jour, sans qu'on puisse parler d une islamisation autoritaire durant sa présidence. Toutefois, Morsi n'a rien fait pour abolir les lois restrictives en matière de médias, héritées du régime de Moubarak. Alors même que les médias privés bénéficiaient d'une grande liberté, rien n'était entrepris dans le secteur des médias d'Etat. Hallin et Mancini ont insisté sur l'importance des médias publics en Europe, pour préciser aussitôt que ceux-ci, loin d'être contrôlés par I'Etat, étaient autonomes et influencés par différentes forces émanant de la société civile. Dès 2011, I'UNESCO a demandé l'abolition des lois restrictives sur les médias et les systèmes d'octroi de licences, et s'est prononcée en faveur de la création d'un secteur public en remplacement des médias d'Etat égyptiens ${ }^{10}$. Durant la présidence de Morsi, des conseillers en médias égyptiens ont travaillé à l'élaboration de plans concrets pour une telle transformation et les ont proposés au gouvernement, mais aucun d'entre eux n'a jamais été adopté et mis en pratique. Au contraire, Morsi a fermement gardé les médias d'Etat sous son contrôle et les a utilisés comme rempart contre les médias privés bien souvent critiques.

La nouvelle Constitution introduite par référendum en novembre 2012 a laissé beaucoup de questions sans réponse concernant le secteur des médias. La Constitution, qui a depuis été abolie, garantissait la liberté de la presse dans la mesure ou la "sécurité nationale», la «mobilisation publique» ou les « insultes aux prophètes de I'islam,

10. Assessment of Media Development in Egypt. Based on UNESCO's Media Development Indicators, UNESCO, Cairo 2011. 
du judaïsme ou du christianisme » ne rendaient pas la censure nécessaire (art. 47 et 48$)^{11}$. Les conditions d'adhésion au "Conseil National des Médias » étaient peu claires, de même que le statut des licences et des autorisations, en particulier, parce que celles-ci étaient délivrées uniquement à ceux qui acceptaient les "valeurs et traditions de la société » (art. 215) - une formulation laissant la porte ouverte à l'interprétation et à la censure du gouvernement. Bien que la Constitution ne fût pas une Constitution islamique entendue au sens de la création d'une dictature islamique comme en Iran, la réglementation des médias restaient vague. Cependant, au lieu d'être abolie, elle aurait pu être amendée, comme cela s'est vu dans plusieurs pays aussi différents que les Etats-Unis et I'Indonésie.

Malgré les imperfections de cette Constitution, la présidence de Morsi a été celle de la stagnation, en non celle de l'islamisation, en matière de médias. Puis le coup d'Etat militaire de juillet 2013 est arrivé. Le CSFA a fermé tous les médias islamistes, mettant fin ainsi à la phase de pluralisme des médias égyptiens. Les bureaux d'Al-Jazeera et de la Corporation de Télévision et de Radio Turque ont été fermés car ils avaient couvert les manifestants des Frères Musulmans. La censure militaire a été rétablie, beaucoup de journalistes ont été tués et plus d'une centaine d'entre eux emprisonnés. Les observateurs parlent $d^{\prime}$ une large coordination entre le CSFA et les médias privés en Egypte ${ }^{12}$.

Avec le passage d'une démocratie semi-libérale à une autocratie de type nassériste, le coup d'Etat militaire a conduit à un retour en arrière. On est passé de la critique du pouvoir politique à la fin de l'ère Moubarak à la liberté débridée sous la présidence Morsi pour finir dans une situation qui évoque ce que William Rugh a appelé les medias " mobilisationalistes » de l'ère Nasser ${ }^{13}$. Sauf qu'à présent, il n'est plus question de "socialisme arabe », mais de lutte contre le "terrorisme islamiste » des Frères Musulmans.

\section{MÉDIAS ET SOCIÉTÉ CIVILE}

Selon Hallin et Mancini, le problème principal dans la relation entre les médias et la société civile est de savoir si les médias prétendent à un " centrisme » libéral ou à un " parallélisme politique ». Dans les jeunes démocraties, les médias sont souvent proches des partis politiques ou

11. Egypt's Draft Constitution Translated, http://www.egyptindependent. com/print/1278681.

12. Kirkpatrick, 18 aout 2013.

13. Rugh, 2004. 
d'autres forces de la société civile. Cependant, la question demeure de savoir s'ils sont capables de couvrir toutes les opinions pertinentes présentes dans la société civile ou s'ils représentent simplement la perspective biaisée d'une élite minoritaire.

Les premières deux années et demi qui ont suivi les révolutions du Printemps arabe étaient caractérisées par un antagonisme politique virulent dans la sphère publique - ce que nous pourrions appelés une "polarisation radicale». Les médias égyptiens étaient "pluralistes» dans la mesure où ils représentaient des points de vue politiques tranchés, mais ils étaient aussi "radicaux » puisqu'ils niaient souvent une quelconque légitimité à l'autre camp. Le clivage le plus décisif existait entre les pro- et les anti-Morsi ou entre pro- et anti-Frères Musulmans.

Les médias pro-Morsi, telles que les chaînes Masr 25, Al-Rahma, Al-Hafez, Al-Nas, Al-Khalijiya, étaient extrêmement partiaux dans leur couverture. Plus d'une fois, ils ont répandu des rumeurs contre les manifestants hostiles à la Constitution de Morsi, affirmant qu'ils étaient saouls et d'autres bruits de ce type. Les médias islamistes du temps de Morsi étaient souvent sectaires et propagandistes. Les téléspectateurs ont craint une islamisation d'autant plus rapide que les médias d'Etat étaient aussi contrôlés par Morsi. Cependant, les médias anti-Morsi n'ont pas fait beaucoup mieux. Le journal Al-Tahrir, comme beaucoup d'autres, a montré une orientation clairement anti-Frères Musulmans - une réalité critiquée par des journalistes au sein même du journal, mais qui $n^{\prime}$ a jamais débouchée sur plus de pluralisme interne ${ }^{14}$. Seul de rares médias, tel qu'Al-Masry Al-Youm, pouvaient prétendre être d'une certaine façon " centristes ».

De plus, il y avait et il y a encore une forte propension à l'adoption d'un « style boulevard » et d'une vision populiste de la politique dans les médias égyptiens. Les médias de masse ont parfois utilisé leur nouvelle liberté pour dénigrer à l'extrême des responsables publics, comme cela s'est vu dans le traitement par le scandale de Mohammed el-Baradei et de sa fille. Bien qu'une analyse au contenu solide soit nécessaire, on peut affirmer sans crainte qu'à la fois la teneur et le ton de beaucoup de médias ne cherchaient pas à trouver un compromis, et encore moins un consensus - principes au coeur de la sphère publique rationnelle d'Habermas, ou de ce que Hallin et Mancini appelle le "média centriste libéral ». Les médias égyptiens rappelaient plutôt ceux de la République de Weimar dans I'Allemagne de l'entre-deux-guerres, avec

14. I am indebted to my PhD-candidate Nadia Leihs for this information. She did numerous interviews in Egyptian newsrooms. 
leurs débats publics polarisés entre des forces politiques intransigeantes ne laissant aucune chance à un soutien constructif au parlement élu et à la démocratisation des institutions. La jeune démocratie allemande était sous la pression de demandes contradictoires, comme ce fut le cas pendant la révolution égyptienne. Beaucoup de gens ont dépassé toute limite dans l'exercice de leurs libertés, après des années de contrainte autoritaire.

D'un point de vue théorique, les médias égyptiens posaient et posent encore beaucoup d'autres problèmes, I'un d'eux étant leur extrême centralisation dans la région du Caire. Alors que des théoriciens comme Hallin et Mancini considèrent comme un point crucial l'idée que la décentralisation régionale a servi de socle au développement des systèmes médiatiques occidentaux démocratiques depuis des siècles, l'Egypte, comme beaucoup d'autres sociétés en développement, souffre $d^{\prime}$ 'une forte concentration dans et autour de la capitale. En conséquence, les problèmes régionaux (le Sud), de même que ceux des paysans, des travailleurs et les intérêts périphériques sont souvent mal représentés. De tels problèmes peuvent aussi être observés dans d'autres pays musulmans. On mentionnera par exemple le cas de l'Indonésie et de la forte concentration des capitaux et des médias dans la région de Jakarta.

Pour résumer, l'atmosphère publique avant et pendant la présidence de Morsi était caractérisée par des problèmes structurels tels que la polarisation radicale de la sphère publique, un manque de pluralisme interne chez la plupart des médias et une intégration insuffisante des problématiques " périphériques » présentes au sein de la société civile.

Cette perspective pourrait ne pas plaire à des théoriciens de la sphère publique comme Mouffe et Laclau, qui plaident au contraire contre l'idée de sphères publiques nationales intégrées. Pour eux, le centralisme libéral rend possible grâce aux médias de masse une forme extrême de contrôle des demandes et de mise en conformité des revendications et des intérêts en provenance de la société. Cependant, Mouffe et Laclau n'ont pas pensé aux jeunes démocraties - le cas de l'Egypte -, qui sont intrinsèquement instables et pour lesquelles l'intégration nationale du discours est absolument nécessaire afin de transformer et de reconstruire les institutions nationales. Habermas semble mieux convenir ici-à moins de considérer qu'Internet représente une alternative viable aux médias de masse?

Durant le Printemps arabe, I'Internet égyptien s'est fortement politisé. Même avant la révolution, l'Egypte était le centre de la blogosphère arabe. Cependant, on I'a dit, le soulèvement égyptien contre Moubarak 
n'a pas été une "révolution Facebook ${ }^{15}$. Seulement dix pour cent des Egyptiens utilisent de tels outils, et les premiers jours du soulèvement, à la fin de janvier 2011, Internet a été coupé par le gouvernement. L'argument selon lequel la coupure $d^{\prime}$ Internet aurait contribué à augmenter le nombre de manifestants est peut-être exact, mais la formule "révolution Facebook » réduit le crédit que méritent d'autres médias - comme Al-Jazeera - et ignorent l'effet de la communication politique directement conduite dans la rue. De la révolution du 25 janvier 2011 jusqu'à aujourd'hui, Internet a certainement permis de combler le déficit d'informations dans les médias de masse. Et à une époque de censure renforcée par l'armée, Internet est devenu d'autant plus important pour n'importe quelle opposition.

Depuis le coup d'Etat militaire de juillet 2013, la polarisation politique, de même que le type de pluralisme qui l'accompagnait, ont pris fin. Nous avons assisté à un retour en arrière vers des médias "mobilisationalistes» et autoritaires de style nassérien. Les médias islamistes ont été fermés et les médias existant montrent un synchronisme dans leur couverture médiatique, et cela jusque dans les moindres détails. Les autorités exercent une énorme pression et la plupart des voix publiques font chorus avec les hyper-nationalistes contre les Frères Musulmans. Les critiques du CSFA sont traités de traîtres. Même les médias qui soutenaient les manifestations Tamarrud et dont on était en droit d'attendre les protestations contre la répression à l'encontre des médias ne le font pas ou ne peuvent pas le faire. La polarisation radicale qui prévalait auparavant ne s'était pas transformée en espace de tolérance entre les groupes, mais celle-ci offrait au moins l'avantage d'être critique du gouvernement et d'agir comme un " quatrième pouvoir ». Avant le coup $d^{\prime}$ Etat militaire, le système médiatique égyptien était comparable au " modèle méditerranéen » de médias démocratiques décrit par Hallin et Mancini, avec un fort secteur gouvernemental et un secteur privé très polarisé. De nos jours, il n'est plus possible de le considérer comme un système démocratique - $c^{\prime}$ est un système autoritaire par nature.

La question se pose alors de savoir dans quelle mesure la polarisation radicale des médias égyptien à l'époque de Morsi a été, au moins partiellement, responsable du climat général tendu qui a pavé la voie à une intervention militaire. Le cas égyptien révèle qu'il n'est pas suffisant de désigner l'émergence d'une sphère publique arabe ${ }^{16}, d^{\prime}$ une " rue arabe ${ }^{17}$, et d'idéaliser les luttes des sociétés civiles arabes contre I'Etat autoritaire.

15. Lynch, 2011.

16. Eickelman et Anderson, 1999.

17. Lynch, 2006. 
Il est grand temps de mettre en avant les faiblesses de la culture politique en Egypte, ainsi que l'incapacité de beaucoup de médias séculiers et islamistes à comprendre que "la liberté est toujours la liberté de celui qui pense différemment», comme l'a dit un jour la célèbre communiste allemande Rosa Luxembourg. La polarisation radicale des sphères publiques dans les jeunes démocraties est peutêtre un phénomène historique largement répandu - mais elle permet aussi de comprendre pourquoi la consolidation de la transformation démocratique est, si souvent, un long et douloureux processus. La forme la plus réussie de transformation démocratique n'est pas la révolution mais réside plutôt dans un " changement de système négocié ${ }^{18}$ entre des forces politiques autrefois radicales qui cherchent à constituer un nouveau consensus.

\section{MÉDIAS ET ÉCONOMIE}

Un des plus grands problèmes de la consolidation démocratique est la concentration du capital dans le secteur des médias. Contrairement à beaucoup de pays d'Amérique latine, le secteur des médias d'Etat dans la plupart des pays arabes demeure considérable. Au début du Printemps arabe, I'UNESCO décrivait les médias égyptiens privés comme très diversifiés du point de vue de leurs propriétaires. ${ }^{19}$ Cependant, il y a une tendance croissante à la concentration des médias et il n'existe pas de contrôle effectif de ce phénomène. Cette concentration a favorisé la réduction du pluralisme dans les médias égyptien. Par exemple, Ibrahim Eissa a dû vendre Al-Tahrir TV à Suleïman Amer, un homme d'affaires lié de près au réseau Moubarak et à I'ancienne élite autoritaire ${ }^{20}$.

L'une des principales propriétés structurelles du système médiatique égyptien provient de ce que l'extrême inégalité dans la distribution du capital propre à la société égyptienne n'a absolument pas été affectée par la révolution. Il est vrai que même les grandes et vieilles démocraties, comme les Etats-Unis, montrent une concentration extrême. La plupart des médias américains sont liés par des structures de propriété et des conseils d'actionnaires à des grands groupes d'affaires. Cela représente un sérieux défi à la liberté des médias. L'idéal type du " média libéral ", évoqué par Hallin et Mancini, n'existe pas. Cependant, lorsque I'on compare I'Egypte aux Etats-Unis, on remarque que le capital aux EtatsUnis n'est pas entièrement "loyaliste" au sens où il ne peut pas être associé à un seul joueur de la scène politique. Le capital investi dans

18. Merkel, 1999.

19. Assessment (UNESCO), op.cit.

20. Sakr, 2013. 
les médias est plus compétitif, si bien que les partis politiques, les institutions sociales et la société civile dans son ensemble sont moins vulnérables qu'en Egypte.

Après des décennies de règne autoritaire et " $d^{\prime}$ Etat profond » en matière financière, toujours organisés autour des réseaux de l'Etat égyptien néo-patrimonial, la dérégulation capitaliste du système médiatique égyptien est une stratégie contestable. II serait certainement plus sage d'opter pour un système double comme en Europe : un secteur réformé des médias publiques, dont l'indépendance et le lien avec la société civile devraient être constitutionnellement garantis, opérant à côté des médias privés capitalistes et des médias alternatifs indépendants.

\section{MÉDIAS ET PROFESSION JOURNALISTIQUE}

Le développement professionnel du journalisme est important pour les médias démocratiques et la sphère publique. Sans formation professionnelle et éthique, on ne peut espérer une amélioration de la qualité des débats publics et une modération adéquate dans leur expression. De plus, une liberté d'exercice de la profession de journaliste dans les salles de rédaction ainsi que des mécanismes professionnels et éthiques d'autorégulation à travers l'existence de syndicats sont des éléments indispensables du journalisme indépendant.

Le syndicat égyptien des journalistes a longtemps été contrôlé par le régime de Moubarak. Ces dernières années, il a été le témoin d'une lutte de pouvoir entre les forces pro et anti-Frères Musulmans. En conséquence, il n'a jamais été capable de se libérer totalement des interférences de l'Etat. Même si le syndicat a protégé les journalistes à diverses reprises, il a aussi toléré la corruption. L'interdiction durable des syndicats indépendants constitue l'un des principaux problèmes en termes d'autorégulation, car il est possible pour ces derniers de bénéficier de se placer sous la protection du syndicat des journalistes. L'adhésion forcée des journalistes est contraire au principe de la libre association qui, comme cela a été mentionné précédemment, est un des aspects essentiels de toute démocratie. Dans sa forme actuelle, l'autorégulation journalistique est, de manière générale, une forme de censure inavouée.

La liberté interne dans les salles de rédaction est un autre sujet sensible en Egypte. Après la révolution, beaucoup de journalistes protestèrent contre leurs rédacteurs en chef (certains furent même contraints de quitter leur poste). Certains médias ont installé de véritables salles de rédaction et renforcé le dialogue interne. Mais la ligne éditoriale et politique de beaucoup de rédacteurs en chef n'a jamais été discutée. 
La situation sociale rend la plupart des journalistes vulnérables aux pressions éditoriales et politiques, voire enclins à la corruption. A l'exception des grosses pointures de la profession, la plupart des journalistes dans les médias privés sont très mal payés. Beaucoup essayent d'obtenir des postes mieux payés dans les médias d'Etat. Pour la majorité des journalistes, il n'y a pas de règles claires en matière de grille salariale, de vacances et de conditions de travail. Sans une plus grande sécurité professionnelle, on ne peut attendre aucune amélioration dans la qualité du journalisme.

\section{CONCLUSION}

Avant le coup d'Etat militaire en juillet 2013, on pouvait dire que I'Egypte avait une sphère publique certes immature et radicalement polarisée, mais vivante. Après le coup d'Etat, cependant, il semble que le pays soit le témoin d'un retour en arrière autoritaire faisant écho au temps de Nasser. Le célèbre intellectuel politique Noam Chomsky a critiqué le coup d'Etat militaire en Egypte : on peut, et on doit, être critique des Frères Musulmans, mais le président Morsi a été légitimement élu. Selon Chomsky, l'armée ne cherche pas à stabiliser la démocratie mais à prendre le pouvoir et à contrôler l'économie ${ }^{21}$. Une fois encore, il semble vrai que les médias de masse soient rarement l'avant-garde de la démocratisation car ils sont trop vulnérables aux manipulations politiques et économiques ${ }^{22}$.

Cependant, les médias et les journalistes sont aussi responsables de la situation actuelle. L'opinion publique radicalement polarisée et échauffée sous la présidence de Morsi, de même que le profond décalage entre les médias islamistes et les médias d'opposition, avec leurs allégeances respectives, ont souvent projeté l'image de ce qu'un politologue libanais a un jour appelé " une démocratie sans démocrate $»^{23}$. L'opinion publique égyptienne, autrefois unie dans un mouvement anti-autoritaire contre Moubarak, a abandonné le consensus et s'est ralliée derrière des camps antagonistes entretenant chacun des positions maximalistes. Il semble que les règles élémentaires de la démocratie ont été ignorées. Alors que les islamistes enfreignaient la notion séculaire d'égalité des religions devant la loi, leurs opposants montraient du mépris pour les élections et le vote du peuple quand celui-ci tournait en faveur de partis, parlement et président islamistes.

21. Chomsky, 17 octobre 2013.

22. McConnell et Becker, 2002.

23. Salamé, 1994. 
Il semble grand temps pour les études des médias dans le monde arabe d'analyser plus en détails à la fois les bénéfices et les échecs des sphères publiques arabes. Les propriétés structurelles de l'Etat, de l'économie, de la société civile et du journalisme professionnel font partie de l'analyse. A présent, la révolution de la communication dans le monde arabe a abouti à une impasse. La réforme est donc plus que jamais nécessaire dans tous les secteurs d'activité. 


\section{BIBLIOGRAPHIE}

Chomsky, N., 17 October 2013, "No one can alienate the Muslim Brotherhood", Middle East Monitor.

EICKelman, D. F., ANDERSON, J. W. (Eds.), 1999, The New Media in the Muslim World. The Emerging Public Sphere, Indiana University Press, Bloomington.

Gramscl, A., 1971, Selection from the Prison Notebooks, International Publishers, New York.

Habermas, J., 1989, The Structural Transformation of the Public Sphere: An Inquiry into a Category of Bourgeois Society, Polity German Orig., Cambridge, 1962.

Heins, M., 1993, Sex, Sin, and Blasphemy. A Guide to America's Censorship Wars, The New Press, New York.

Hafez, K., 1996, "The Algerian Crisis as Portrayed in the German Press: Media Coverage of Political Islam", Communications. The European Journal of Communication Research, 21 (2), p. 155-182.

Hafez, K., 2010, Radicalism and Political Reform in the Islamic and Western World, Cambridge University Press, Cambridge.

Hallin, D. C., MancinI, P., 2004, Comparing Media Systems; The three Models of Media and Politics, Cambridge University Press, Cambridge.

Herman, E. S., Chomsky, N., 1988, Manufacturing Consent, Pantheon Books, New York.

Khamis, S., 2009, "Modern Egyptian Media: A Transitional Press Model in a Comparative Context, Paper presented at International Communication Association Conference, Chicago. KIRKPATRICK, D. D., 18 August 2013, "Egypt Lashes out at Foreign News Media's Coverage", The New York Times.

Laclau, E., Mouffe, C., 2001, Hegemony and Socialist Strategy. Towards a Radical Democratic Politics, London, Verso.

LYNCH, M., 2006, Voices of the New Arab Public. Iraq, Al-Jazeera, and Middle East Politics Today, Columbia University Press, New York.

LyNCH, M., 2011, "After Egypt: The Limits and Promise of Online Challenges to the Authoritarian Arab State", Perspectives on Politics, 9 (2), p. 301-310.

Mcconnel, P. J., Becker, L. B., 2002, "The Role of the Media in Democratization", Paper Presented to the Political Communication Section of the International Association for Media and Communication Research at the Barcelona Conference (http:// www.grady.uga.edu/coxcenter/activities/Act_2001_to_2002/ Materials01-02/DemocratizationIAMCRJuly2002.pdf).

Merkel, W., 1999, Systemtransformation. Eine Einführung in die Theorie und Empirie der Transformationsforschung [System Transformation. An Introduction to the Theory and Empirical Practice of Research on Political Transformation], Leske \& Budrich, Opladen. 
Rugh, W. A., 2004, Arab Mass Media. Newspapers, Radio, and Television in Arab Politics, Praeger, London.

SAKR, N., 2013, Transformations in Egyptian Journalism, I.B. Tauris, London.

Salame, G. (ed.), 1994, Democracy without Democrats? The Renewal of Politics in the Muslim World, I.B. Tauris, London. 